\title{
Chondrocalcinosis is common in the absence of knee involvement
}

\author{
Abhishek Abhishek ${ }^{1 *}$, Sally Doherty ${ }^{1}$, Rose Maciewicz ${ }^{2}$, Kenneth Muir ${ }^{3}$, Weiya Zhang ${ }^{1}$ and Michael Doherty ${ }^{1}$
}

\begin{abstract}
Introduction: We aimed to describe the distribution of radiographic chondrocalcinosis (CC) and to examine whether metacarpophalangeal joint (MCPJ) calcification and CC at other joints occurs in the absence of knee involvement.
\end{abstract}

Methods: This was a cross-sectional study embedded in the Genetics of Osteoarthritis and Lifestyle study (GOAL). All participants $(n=3,170)$ had radiographs of the knees, hands, and pelvis. These were scored for radiographic changes of osteoarthritis (OA), for CC at knees, hips, symphysis pubis, and wrists, and for MCPJ calcification. The prevalence of MCPJ calcification and CC overall, at each joint, and in the presence or absence of knee involvement, was calculated.

Results: The knee was the commonest site of CC, followed by wrists, hips, and symphysis pubis. CC was more likely to be bilateral at knees and wrists but unilateral at hips. MCPJ calcification was usually bilateral, and less common than CC at knees, hips, wrists, and symphysis pubis. Unlike that previously reported, CC commonly occurred without any knee involvement; $44.4 \%$ of wrist CC, $45.9 \%$ of hip CC, $45.5 \%$ of symphysis pubis CC, and $31.3 \%$ of MCPJ calcification occurred in patients without knee CC. Those with meniscal or hyaline articular cartilage CC had comparable ages $(P=0.21)$, and neither preferentially associated with fibrocartilage $C C$ at distant joints.

Conclusions: CC visualized on a plain radiograph commonly occurs at other joints in the absence of radiographic knee CC. Therefore, knee radiographs alone are an insufficient screening test for CC. This has significant implications for clinical practice, for epidemiologic and genetic studies of CC, and for the definition of OA patients with coexistent crystal deposition.

\section{Introduction}

Chondrocalcinosis $(\mathrm{CC})$ predominantly results from calcium pyrophosphate (CPP) crystal deposition (CPPD) [1]. Although the knee is the commonest location of CC, it is unclear whether the wrist [1-4], or the symphysis pubis is the second most commonly affected joint [5-7]. Similarly, the frequency with which CC occurs at other joints in the absence of knee involvement is unclear. Most studies suggest that $\mathrm{CC}$ occurs uncommonly or does not occur in the absence of knee involvement [1,3,4,6-10]. For example, a study using a complete skeletal survey of 48 participants suggested that hand, knee, and pelvis radiographs together were sufficient to identify patients with $\mathrm{CC}$ at any joint [1]. It also suggested that $89 \%$ of cases with $\mathrm{CC}$ at any site

\footnotetext{
* Correspondence: docabhishek@gmail.com

${ }^{1}$ Academic Rheumatology, University of Nottingham, Nottingham NG5 1PB, UK

Full list of author information is available at the end of the article
}

could be identified by using knee radiographs, and that this figure increased to $98 \%$ when both knees and pelvis were radiographed [1]. This observation promoted a notion that knee radiographs alone may be a sufficient screening test to identify most patients with CC. However, a couple of relatively small studies suggested that one fourth to one third of all CC cases did not have any knee involvement $[2,5]$.

Establishing whether CC commonly occurs in those without knee involvement is important, as it will facilitate the diagnosis of arthropathy associated with CPPD, and will allow accurate ascertainment of cases and controls for research. The overall aim of this study was to examine the distribution of CC by using knee, pelvis, and hand radiographs. The objectives were to calculate the prevalence of $\mathrm{CC}$ at each joint and of calcification in the synovium and capsule of metacarpophalangeal joints (MCPJs), which associates with CC [11]; to compare the

\section{() Biomed Central}


prevalence of $\mathrm{CC}$ at other joints and the prevalence of $\mathrm{MCPJ}$ calcification in the presence and absence of knee $\mathrm{CC}$; and to examine whether fibrocartilage $\mathrm{CC}$ at a distant joint preferentially occurs in those with meniscal or hyaline articular cartilage $\mathrm{CC}$ at the knee.

\section{Materials and methods}

This cross-sectional study used data from the Genetics of Osteoarthritis and Lifestyle (GOAL) study. GOAL comprises 3,170 individuals: 1,007 with clinically severe hip OA, 1,042 with clinically severe knee OA, and 1,121 without knee or hip OA, recruited from 2002 to 2006. The study was approved by the Nottinghamshire Research Ethics Committee, UK.

The details of GOAL have been published elsewhere [12]. In brief, all participants completed a detailed questionnaire, were examined by a research metrologist, gave urine and blood samples, and underwent the following standardized radiographic assessments: posteroanterior weight-bearing semiflexed knee radiographs by using the SynaFlexer positioning frame (Synarc); skyline views of patellofemoral joints; supine pelvis radiographs for hips and symphysis pubis; and anteroposterior hand views, which included the wrists.

Radiographs were graded for CC at the knees, hips, wrists, and symphysis pubis, for MCPJ calcification, and for structural changes of $\mathrm{OA}$ by a senior research metrologist (SD). CC was present if linear or nummular calcification in fibro- or hyaline articular cartilage was found; synovial/capsular calcification (occasionally also $\mathrm{CC})$ was also recorded at the metacarpophalangeal joints (MCPJs). Knee CC was scored for compartmental (lateral or medial tibiofemoral joint (TFJ) compartment), and cartilaginous location (hyaline or fibrocartilage).

\section{Statistical analysis}

Mean (standard deviation (SD)) and $n$ (\%) were used to describe continuous and categoric variables. Analysis of variance (ANOVA) was used to compare continuous variables in different categories. The prevalence and 95\% confidence interval (CI) of CC (including MCPJ calcification) was calculated at each joint region for any, unilateral, bilateral, and isolated CC (that is, involvement of target joint without involvement of any distant joint).

The $95 \%$ CI was calculated as $p \pm 1.96 \times \sqrt{\frac{p(1-p)}{n}}$, where $p$ is the prevalence and $n$ is the number of participants. The prevalence ( $95 \% \mathrm{CI}$ ) of $\mathrm{CC}$ and MCPJ calcification at other joints in the presence or absence of knee CC also was calculated. The prevalence of symphysis pubis $\mathrm{CC}$, a marker of fibrocartilage calcification, was compared between those with isolated meniscal CC, isolated hyaline cartilage $\mathrm{CC}$, and both meniscal and hyaline articular cartilage $\mathrm{CC}$ at the knee. Statistical analysis was done by using SPSS v14.

\section{Results}

The mean (SD) age of participants was 66.6 (7.9) years, and 1,536 (48.5\%) were women. Radiographic knee OA and hip OA were present in 1,602 (51.0\%) and 1,168 (36.9\%) participants. Information about CC and MCPJ calcification at all joints radiographed was available for 3,118 participants who were included in the analysis.

\section{Distribution of chondrocalcinosis}

In total, 428 participants had MCPJ calcification or CC at any site (prevalence (95\% CI) $13.7 \%$ (12.6\% to $15.0 \%)$ ). Knee was the commonest site involved (Table 1). The prevalence $(95 \% \mathrm{CI})$ of CC at any knee, hip, or symphysis pubis; and of $\mathrm{CC}$ at any knee, wrist, or MCPJ calcification was $11.3 \%$ (10.3\% to $12.4 \%)$, and $11.2 \%$ (10.1\% to $12.3 \%)$, respectively. MCPJ calcification most commonly occurred at the index and middle fingers. $\mathrm{CC}$ at other joints and MCPJ calcification were common in the absence of knee involvement (Table 2). In participants with CC at any site or MCPJ calcification, only $58.4 \%$ (250 of 428), $52.3 \%$ (228 of 428), and $48.4 \%$ (207 of 428) could be detected by using knee, hand, and pelvis radiographs alone (Figure 1a). This increased to $82.5 \%$ (355 of 428 ), $81.5 \%$ (349 of 428), and $80.1 \%$ (343 of 428 ) when knee and pelvis, knee and hand, and hand and pelvis radiographs were examined in combination (Figure 1a).

As GOAL participants have a high prevalence of hip or knee OA, and OA can affect the distribution of CC, data about distribution of CC were analyzed for 1,109 GOAL participants without radiographic hip or knee OA for whom CC could be scored at all joints examined. Eightythree participants had MCPJ calcification or CC at any site. In this subset, only $56.7 \%$ (47 of 83 ), $28.9 \%$ (24 of 83 ), and $65.1 \%$ (58 of 83) participants with CC at any joint or MCPJ calcification could be detected on knee, hand, and pelvis radiographs alone (Figure $1 \mathrm{~b}$ ).

\section{Knee chondrocalcinosis}

Knee CC was more common in the lateral TFJ compartment than in the medial TFJ compartment (right: $90.9 \%$ lateral, $76.1 \%$ medial; left: $87.2 \%$ lateral, $71.8 \%$ medial; $P<0.001)$. It affected the fibrocartilaginous meniscus more frequently than the intraarticular hyaline cartilage (right: 91.4\% fibro-, 56.8\% hyaline; left: $86.3 \%$ fibro-, $53.7 \%$ hyaline; $P<0.001)$. Similarly, isolated fibrocartilage involvement was more common than isolated hyaline cartilage involvement (right knee: $42.8 \%$ fibro-, $8.2 \%$ hyaline; left knee: $46.3 \%$ fibro-, $13.7 \%$ hyaline; $P<$ 0.001). At the knee, patients with isolated meniscal CC, isolated hyaline articular cartilage $\mathrm{CC}$, and $\mathrm{CC}$ in both fibro- and hyaline articular cartilage were of comparable 
Table 1 Prevalence of chondrocalcinosis (CC) and metacarpophalangeal joint (MCPJ) calcification

\begin{tabular}{lllllll}
\hline Index joint & \multicolumn{7}{l}{ Prevalence $(\mathbf{9 5 \%}$ Cl) of CC } \\
\cline { 2 - 7 } & Overall (\%) & Right (\%) & Left (\%) & Unilateral (\%) & Bilateral (\%) & Isolated (\%) \\
\hline Knee & $8.0(7.0-8.9)$ & $6.7(5.8-7.5)$ & $6.0(5.2-6.8)$ & $3.3(2.7-3.9)$ & $4.7(4.0-5.4)$ & $2.7(2.2-3.4)$ \\
Wrist & $6.9(6.0-7.8)$ & $5.4(4.6-6.2)$ & $5.8(5.0-6.6)$ & $2.7(2.1-3.2)$ & $4.3(3.6-4.9)$ & $2.0(1.6-2.6)$ \\
Hip & $5.0(4.3-5.9)$ & $4.0(3.3-4.7)$ & $3.0(2.4-3.6)$ & $3.1(2.5-3.7)$ & $2.0(1.5-2.5)$ & $1.4(1.0-1.9)$ \\
Symphysis & $3.6(2.9-4.2)$ & N/A & N/A & N/A & N/A & $0.8(0.5-1.2)$ \\
Any MCPJs & $1.5(1.1-2.0)$ & $0.9(0.6-1.2)$ & $1.1(0.7-1.4)$ & $0.4(0.2-0.6)$ & $1.1(0.8-1.5)$ & $0.1(0.0-0.3)$ \\
\hline
\end{tabular}

age, with a mean (SD) age of 69.7 (6.7), 69.5 (7.4), and 71.0 (5.4) years, respectively $(P=0.21)$.

\section{Symphysis pubis chondrocalcinosis}

Of the 3,131 participants for whom information about knee and symphysis pubis CC was available, isolated hyaline cartilage $\mathrm{CC}$, isolated meniscal $\mathrm{CC}$, and both meniscal and hyaline cartilage CC at the knee were present in 20, 97, and 130 participants, respectively; and the prevalence (95\% CI) of symphysis pubis CC in these groups was $10 \%$ (2.8\% to $30.0 \%$ ), $25.8 \%$ (18.1\% to $35.3 \%$ ), and $26.2 \%$ (19.4\% to $34.3 \%$ ), respectively. Knee CC was absent in 2,884 participants, and $1.8 \%$ (1.4\% to $2.3 \%$ ) of these had symphysis pubis CC.

\section{Discussion}

This is the first large study to examine the distribution of $\mathrm{CC}$ and MCPJ calcification by using knee, hand, and pelvis radiographs $[1,5,6]$. We report that hip, wrist, and symphysis pubis CC and MCPJ calcification commonly occur without knee involvement. We also found that wrist $\mathrm{CC}$ is more common than hip CC, and that CC is more likely to be bilateral, except at the hips. We confirmed previous observations that the lateral TFJ compartment $[5,9,13,14]$ and the menisci are the preferred sites for knee CC $[2,5,7,8,11]$.

In our study, $42 \%$ of the CC cases had no knee involvement. This suggests that a significant proportion of cases with $\mathrm{CC}$ would be missed if knee radiographs alone were used to screen for $\mathrm{CC}$, and this would result in important misclassification (for example, in genetic case-control studies). Although similar findings have been reported in two small studies [2,5], this is discordant with most previous reports $[1,3,4,6-10,15]$. Previous studies were small, hospital based, and were therefore likely to overrepresent symptomatic knee arthropathy cases. Similarly, in our study, only $80 \%$ to $82 \%$ of cases with CC at any site or $\mathrm{MCPJ}$ calcification could be identified by using radiographs of any two regions alone. This suggests that radiographs of knees, hips, and hands should be performed to screen adequately for $\mathrm{CC}$, and is not in keeping with a previous study that suggested that $98 \%$ of cases with CC could be identified by using pelvis and knee radiographs alone [1].

Despite a high prevalence of OA, the prevalence of CC at knees, knees and pelvis, and knees and wrists reported here are similar to those in community studies $[9,14,15]$. GOAL participants have a high prevalence of large-joint OA and would be expected to have a twofold greater prevalence of CC [14]. However, they were also approximately 10 years younger than those in population studies of CC $[9,14,15]$. Because the prevalence of CC halves for each 10-year decrease in age, this probably compensated for any increase in prevalence of CC resulting from a high prevalence of OA [14].

Because this is the largest study to compare the prevalence of $\mathrm{CC}$ at wrists and hips, we can infer that the wrist is the second most common site for CC. In our study, CC was more common at the hips than at the symphysis pubis [5-7]. However, in one large community-based survey, symphysis pubis calcification was 10 -fold more common than hip CC [9]. The difference in prevalence between this survey and our study may again result from the high prevalence of severe symptomatic hip or knee $\mathrm{OA}$ and the younger age of GOAL participants, which might increase the prevalence of $\mathrm{CC}$ at the hips but not at the symphysis pubis.

As reported previously, no predilection was found for $\mathrm{CC}$ to occur on any side [14]. However, a study reported

Table 2 Chondrocalcinosis (CC) at other sites and metacarpophalangeal joint (MCPJ) calcification in the presence or absence of knee involvement

\begin{tabular}{llll}
\hline Index joint & $\begin{array}{l}\text { Knee CC } \\
+ \text { ve }\end{array}$ & $\begin{array}{l}\text { Knee CC } \\
\text {-ve }\end{array}$ & Proportion (95\%Cl) with CC at index joint without knee involvement \\
\hline Wrist & 120 & 96 & $44.4(38.0-51.0) \%$ \\
Hip & 85 & 72 & $45.9(38.3-53.4) \%$ \\
Symphysis pubis & 61 & 51 & $45.5(36.6-54.8) \%$ \\
Any MCPJs & 33 & 15 & $31.3(20.0-45.0) \%$ \\
\hline
\end{tabular}



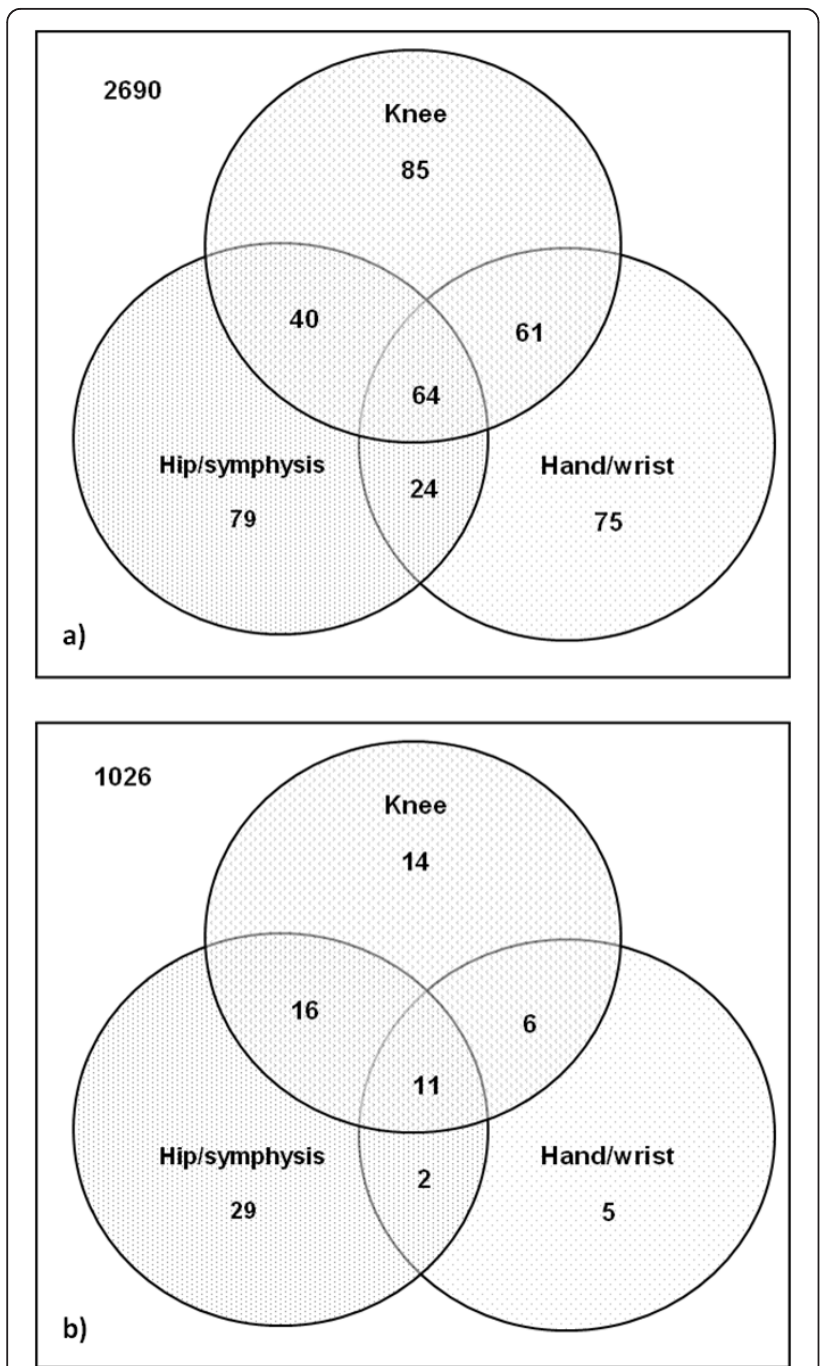

Figure 1 Distribution of CC and metacarpophalangeal joint (MCPJ) calcification in knee, hand, and pelvis radiographs in (a) all participants $(n=3118)$, and in (b) participants without knee or hip OA $(n=1,109)$.

unilateral CC preferentially affecting the right knee [9]. In agreement with other reports, $\mathrm{CC}$ was more likely to be bilateral at the knees and wrist $[2-5,8,9,11,14]$. However, unlike prior studies, CC was likely to be unilateral at the hips $[2,4,5,8,11]$. More than $90 \%$ of cases with MCPJ calcification had CC at other joints, suggesting that MCPJ calcification is part of CPPD. Similar findings have been reported before [11].

Patients with isolated meniscal $\mathrm{CC}$, isolated hyaline cartilage $\mathrm{CC}$, and $\mathrm{CC}$ in both meniscus and hyaline cartilage of the knee were of similar age. This suggests that knee CC occurs at the same time in both fibro- and hyaline articular cartilage. However, this should be confirmed in a community-based survey to exclude any effect of healthcare seeking bias. Moreover, fibrocartilage
$\mathrm{CC}$ at a distant joint (for example, symphysis pubis) was not significantly more common in those with isolated meniscal $\mathrm{CC}$, or $\mathrm{CC}$ at both meniscus and hyaline articular cartilage than in those with isolated hyaline cartilage $\mathrm{CC}$. We were unable to examine whether hyaline cartilage $\mathrm{CC}$ at the hip is more common in those with hyaline cartilage $\mathrm{CC}$ at the knee, because confident specification of tissue localization of CC at the hip is difficult by using a single-view plain radiograph. Further macroradiographic studies are warranted to examine the prevalence of fibro- and hyaline articular cartilage $\mathrm{CC}$ at the hip, and to examine their relation with fibro- and hyaline articular cartilage CC at the knee.

This is the largest study to examine the distribution of $\mathrm{CC}$ and MCPJ calcification. Several caveats to these findings exist. First, GOAL was designed as a case-control cohort to examine risk factors for large-joint OA. In the present study, we undertook a nested study that involved reattribution of case and control status. Two thirds of participants had severe symptomatic hip or knee OA, and the remaining have no radiographic or clinical features of hip or knee OA. The population under study therefore limits the generalizability of the findings. However, the prevalence of CC at the knee, knee and hip/ symphysis pubis, knee and wrist reported in this study are similar to those observed in large community-based radiographic surveys $[9,14,15]$, providing face validity. Moreover, a similar pattern was observed when data for patients without large-joint OA were analyzed. Finally, it is possible that knee CC detectable on ultrasound may be present in some participants who do not have visible CC on knee radiographs and have $\mathrm{CC}$ at other joints. However, plain radiographs are still the first-line radiographic examination in patients with arthropathy, and in routine clinical practice, not all patients undergo musculoskeletal ultrasound. The results of our study are therefore transferable to routine clinical practice, and to large epidemiologic studies of CC.

\section{Conclusions}

$\mathrm{CC}$ at other joints and MCPJ calcification are common in the absence of knee involvement, and do not prefer one side over the other. In those with CC, wrist is the second most commonly involved site after the knee. Knee CC, wrist CC, and MCPJ calcification, but not hip CC, are more often bilateral. At the knee, $\mathrm{CC}$ is more common at the lateral TFJ compartment than in the medial TFJ compartment; and in the menisci than in hyaline cartilage. There was no evidence to suggest that either meniscal or hyaline articular cartilage CC precedes the other, or preferentially associates with fibrocartilage $\mathrm{CC}$ at distant joints. These findings derived from a hospital-based population should be confirmed in a general populationbased cross-sectional study. 


\section{Abbreviations}

ANOVA: analysis of variance; CC: chondrocalcinosis; Cl: confidence interval; CPP: calcium pyrophosphate crystal; CPPD: calcium pyrophosphate crystal deposition; GOAL: genetics of osteoarthritis and lifestyle; MCPJ:

metacarpophalangeal joint; $n$ : number of participants; OA: osteoarthritis; $p$ : prevalence; SD: standard deviation; TFJ: tibiofemoral joint.

\section{Acknowledgements}

We are grateful to AstraZeneca, Macclesfield, UK for financial support for the GOAL study and to Arthritis Research UK for infrastructure support during assembly of the GOAL cohort (ICAC grant 14851).

\section{Author details}

${ }^{1}$ Academic Rheumatology, University of Nottingham, Nottingham NG5 1PB, UK. ${ }^{2}$ Respiratory and Inflammation iMed, AstraZeneca, Mölndal, Sweden. ${ }^{3}$ Health Sciences Research Institute, University of Warwick, Warwick CV4 7AL, UK.

\section{Authors' contributions}

AA was involved in study conception and design and in analysis and interpretation of the data. SD was involved in study conception, data acquisition, and radiographic scoring. $\mathrm{RM}, \mathrm{KM}, \mathrm{WZ}$, and $\mathrm{MD}$ were involved in study conception and design and in analysis and interpretation of the data. $\mathrm{KM}$ and MD were involved in data acquisition as well. All authors were involved in drafting the article or revising it, and all authors approved the final version.

\section{Competing interests}

AstraZeneca had no role in the study design, data analysis, and in drafting of this manuscript. Dr. Rose Maciewicz is an employee of AstraZeneca, owns shares in that company, and was involved as a scientific collaborator in the GOAL study. Other authors have no competing interests to declare.

Received: 21 May 2012 Revised: 11 August 2012

Accepted: 4 October 2012 Published: 4 October 2012

\section{References}

1. Resnick D, Niwayama G, Goergen TG, Utsinger PD, Shapiro RF, Haselwood DH, Wiesner KB: Clinical, radiographic and pathologic abnormalities in calcium pyrophosphate dihydrate deposition disease (CPPD): pseudogout. Radiology 1977, 122:1-15.

2. McCarty DJ Jr, Haskin ME: The roentgenographic aspects of pseudogout (articular chondrocalcinosis). an analysis of 20 cases. Am J Roentgenol Radium Ther Nucl Med 1963, 90:1248-1257.

3. Louthrenoo W, Sukitawut W: Calcium pyrophosphate dihydrate crystal deposition: a clinical and laboratory analysis of 91 Thai patients. J Med Assoc Thai 1999, 82:569-576.

4. Ellman MH, Levin B: Chondrocalcinosis in elderly persons. Arthritis Rheum 1975, 18:43-47.

5. Wilkins E, Dieppe P, Maddison P, Evison G: Osteoarthritis and articular chondrocalcinosis in the elderly. Ann Rheum Dis 1983, 42:280-284.

6. Gordon TP, Smith M, Ebert B, McCredie M, Brooks PM: Articular chondrocalcinosis in a hospital population: an Australian experience. Aust N Z J Med 1984, 14:655-659.

7. Fam AG, Topp JR, Stein HB, Little AH: Clinical and roentgenographic aspects of pseudogout: a study of 50 cases and a review. Can Med Assoc J 1981, 124:545-551.

8. Zitnan D, Sitaj S: Chondrocalcinosis articularis, Section I: Clinical and radiological study. Ann Rheum Dis 1963, 22:142-152.

9. Ramonda R ME, Perissinotto E, Sartori L, Punzi L, Corti MC, Hirsch R, Manzato E, Zambon S, Baggio G, Crepaldi G: Prevalence of chondrocalcinosis in Italian subjects from northeastern Italy: the Pro.V.A. (PROgetto Veneto Anziani) study. Clin Exp Rheumatol 2009, 27:4.

10. Canhao H, Fonseca JE, Leandro MJ, Romeu JC, Pimentao JB, Costa JT, Queiroz MV: Cross-sectional study of 50 patients with calcium pyrophosphate dihydrate crystal arthropathy. Clin Rheumatol 2001, 20:119-122.

11. Genant HK: Roentgenographic aspects of calcium pyrophosphate dihydrate crystal deposition disease (pseudogout). Arthritis Rheum 1976, 19(Suppl 3):307-328.
12. Robertson J, Zhang W, Liu JJ, Muir KR, Maciewicz RA, Doherty M: Radiographic assessment of the index to ring finger ratio (2D:4D) in adults. J Anat 2008, 212:42-48.

13. Riestra JL, Sanchez A, Rodriques-Valverde V, Castillo E, Calderon J: Roentgenographic features of the arthropathy associated with CPPD crystal deposition disease: a comparative study with primary osteoarthritis. J Rheumatol 1985, 12:1154-1158.

14. Felson DT, Anderson JJ, Naimark A, Kannel W, Meenan RF: The prevalence of chondrocalcinosis in the elderly and its association with knee osteoarthritis: the Framingham Study. J Rheumatol 1989, 16:1241-1245.

15. Sanmarti RP, Brancos D, Canela MA, Collado J, Brugues J: Prevalence of articular chondrocalcinosis in elderly subjects in a rural area of Catalonia. Ann Rheum Dis 1993, 52:418-422.

doi:10.1186/ar4043

Cite this article as: Abhishek et al:: Chondrocalcinosis is common in the absence of knee involvement. Arthritis Research \& Therapy 2012 14:R205.

\section{Submit your next manuscript to BioMed Central and take full advantage of:}

- Convenient online submission

- Thorough peer review

- No space constraints or color figure charges

- Immediate publication on acceptance

- Inclusion in PubMed, CAS, Scopus and Google Scholar

- Research which is freely available for redistribution

Submit your manuscript at www.biomedcentral.com/submit
Ciomed Central 